\section{Relationship between peripheral visual field loss and vision-related quality of life in patients with retinitis pigmentosa}

T Sugawara, A Hagiwara, A Hiramatsu, K Ogata, Y Mitamura and S Yamamoto

\begin{abstract}
Purpose To determine the vision-related quality of life (VRQOL) with the National Eye Institute Visual Function Questionnaire-25 (NEI VFQ-25) in patients with retinitis pigmentosa (RP), and to examine the relationship between VRQOL and peripheral visual field defects. Design Prospective study.

Methods The Japanese version of the NEI VFQ-25 was used to study 40 patients with typical RP whose visual acuity was $\geqslant 0.7$ (better than 0.15 in $\log$ MAR). For control, 40 volunteers with normal vision were studied in the same way. The peripheral visual field was evaluated by Goldmann's perimetry, and the degree of field loss was classified into seven grades. The correlation between the mean of the total composite score of the NEI VFQ-25 and the degree of the visual field loss was determined.
\end{abstract}

Results The mean NEI VFQ-25 score was 68.4 in RP patients and $\mathbf{9 0 . 1}$ in normal controls. This difference was highly significant $(P=0.00004)$. Among RP patients, there was a significant negative correlation between the mean NEI VFQ-25 score and the degree of visual field loss $(r=-0.519, P=0.0006)$. Conclusion The significant correlation between the peripheral visual field loss and VRQOL score obtained with the NEI VFQ-25, indicates that a good estimate of the QOL can be determined by the degree of visual field loss in RP patients.

Eye (2010) 24, 535-539; doi:10.1038/eye.2009.176; published online 10 July 2009

Keywords: retinitis pigmentosa; quality of life; NEI VFQ-25; Goldmann's perimetry

\section{Introduction}

Retinitis pigmentosa (RP) is one of the leading causes of blindness, and is characterized by night blindness, progressive visual field defects, and impairment of central vision. The visual acuity, visual fields (perimetry), and electroretinograms (ERGs) have been used to assess the visual function of RP patients in routine clinical tests. The ERGs are markedly reduced or non-recordable in most RP patients even at the early stage. However, other assessments are necessary for a more detailed evaluation on how the depressed visual function affects the daily living of RP patients.

The importance of evaluating the outcomes of health care on patients is now widely realized to be very important. Thus, there have been several studies that have evaluated the performance of daily activities by RP patients. ${ }^{1-3}$ These studies, which used the investigators' questionnaire, found that the visual acuity and visual fields mostly influenced the patients' daily life.

Recently, a National Eye Institute Visual Function Questionnaire-25 (NEI VFQ-25) was developed from the NEI VFQ-51 to test the psychometric properties of those diseases that cause vision decrease to evaluate the visionrelated quality of life (VRQOL). ${ }^{4,5}$ This questionnaire with 25 questions has been reported to provide reliable and valid scores of patients with a variety of eye diseases. ${ }^{6-11}$ The NEI VFQ-25 has been translated into other languages, ${ }^{12,13}$ and its usefulness in assessing the VRQOL in Japanese patients with cataract, glaucoma, and age-related macular degeneration (AMD) has been reported. ${ }^{14}$
Department of Ophthalmology and Visual Science, Chiba University Graduate School of Medicine, Chiba, Japan

Correspondence:

T Sugawara, Department of Ophthalmology and Visual Science, Chiba University Graduate School of Medicine, 1-8-1 Inohana, Chuo-ku, Chiba 260-8670, Japan

Tel: + 81432262124 ;

Fax + 8143224 4162;

E-mail: takeshis@

faculty.chiba-u.jp

Received: 12 November 2008

Accepted in revised form: 11 June 2009 Published online: 10 July 2009 
There have been several reports using the NEI VFQ-25 to examine the QOL of patient with different types of retinal dystrophies. ${ }^{11,15,16} \mathrm{Hahm}$ et al., ${ }^{11}$ reported that RP patients with mental depression had poorer visionrelated functions compared with those without depression. They also reported that the poorer vision-related functions could not be explained by the visual acuity.

The purpose of this study was to evaluate the VRQOL in RP patients using the NEI VFQ-25, and to investigate the relationship between the scores of the NEI VFQ-25 and the visual field loss in RP patients who had relatively good visual acuity $(\geqslant 0.7)$.

\section{Materials and methods}

\section{Patients}

In all, 40 consecutive patients ( 20 men and 20 women) with bilateral typical RP were studied at the Eye Clinic of the Chiba University Hospital from November 2006 to February 2007. The mean age of the patients was $50.9 \pm 13.5$ years with a range from 20 to 71 years. RP patients whose best-corrected visual acuity (BCVA) was $\geqslant 0.7$ (0.15 logMAR units) were studied. To evaluate the influence of the peripheral visual field loss on the VRQOL, only patients with good preservation within the central $10^{\circ}$, but had different degrees of peripheral visual field defects, were studied. Patients with cataract, cystoid macular oedema, or other ocular diseases

causing visual impairment were excluded. A total of 40 age-matched normal volunteers, who were doctors and nurses, served as controls.

The study protocol adhered to the tenets of the Declaration of Helsinki. The purpose and procedures to be used in this study were carefully explained to all patients and control subjects, and an informed consent was obtained from all.

\section{NEI VFQ-25}

Patients answered the Japanese version of NEI VFQ-25 by themselves by following the protocol of Suzukamo, who developed the Japanese version of the questionnaire. ${ }^{14}$ All patients were able to read and complete the questionnaires without an interviewer because all had good central vision and understood the questions.

The NEI VFQ-25 is made up of 25 questions that addressed 12 aspects of daily living: general health, general vision, near vision, distance vision, driving, peripheral vision, colour vision, ocular pain, role limitation, dependency, social function, and mental health. We excluded general health and driving because it has been reported that the general health was not directly related with visual condition, ${ }^{14}$ and most RP patients who were enrolled had already given up driving because of their visual impairments. The answer to each of the 25 questions was converted to a 100-point scale, in which 100 represents the best possible score and 0 represents the worst score, and each aspect represented the average of one or more questions. The composite VFQ-25 score was the mean score of some subscales. ${ }^{14}$

\section{Classification of peripheral visual field loss}

The visual fields were determined by Goldmann's perimetry monocularly. The degree of visual field loss was classified into seven grades by the V4e indicator according to the reported classifications for RP patients, ${ }_{1}^{17-19}$ which were slightly modified. The visual field loss was graded from 0 to 6 : grade 0 , normal visual field; grade 1, some scattered scotomas in the midperiphery; grade 2, ring scotoma; grade 3, constricted visual field within the central $30^{\circ}$; grade 4 , constricted visual field within the central $15^{\circ}$ with isolated peripheral visual islands; grade 5 , constricted visual field within the central $15^{\circ}$ without peripheral visual islands; and grade 6 , constricted visual field within the central $10^{\circ}$ (Figure 1). The grading of the visual field was done by two of the authors (TS and $\mathrm{KO}$ ) who were blinded to other information about the patient at the time of grading. Patients whose visual field did not fit into this classification, and patients whose classification was not the same for the two observers were removed from the study.

The average NEI VFQ-25 scores were compared between RP patients and normal controls using Mann-Whitney $U$-test. The correlation between the
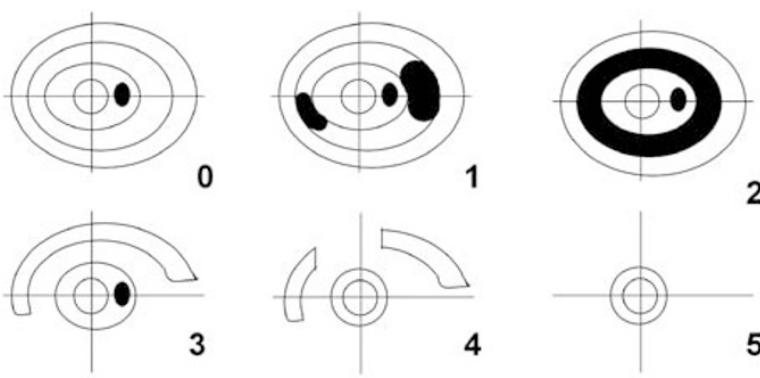

2

4
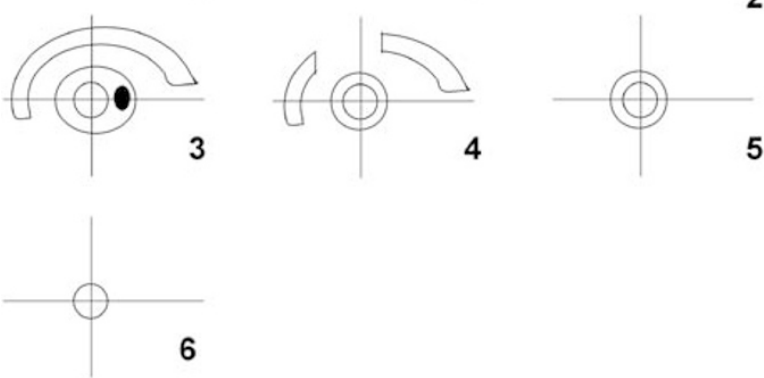

Figure 1 Classification of visual field defects in retinitis pigmentosa patients. 

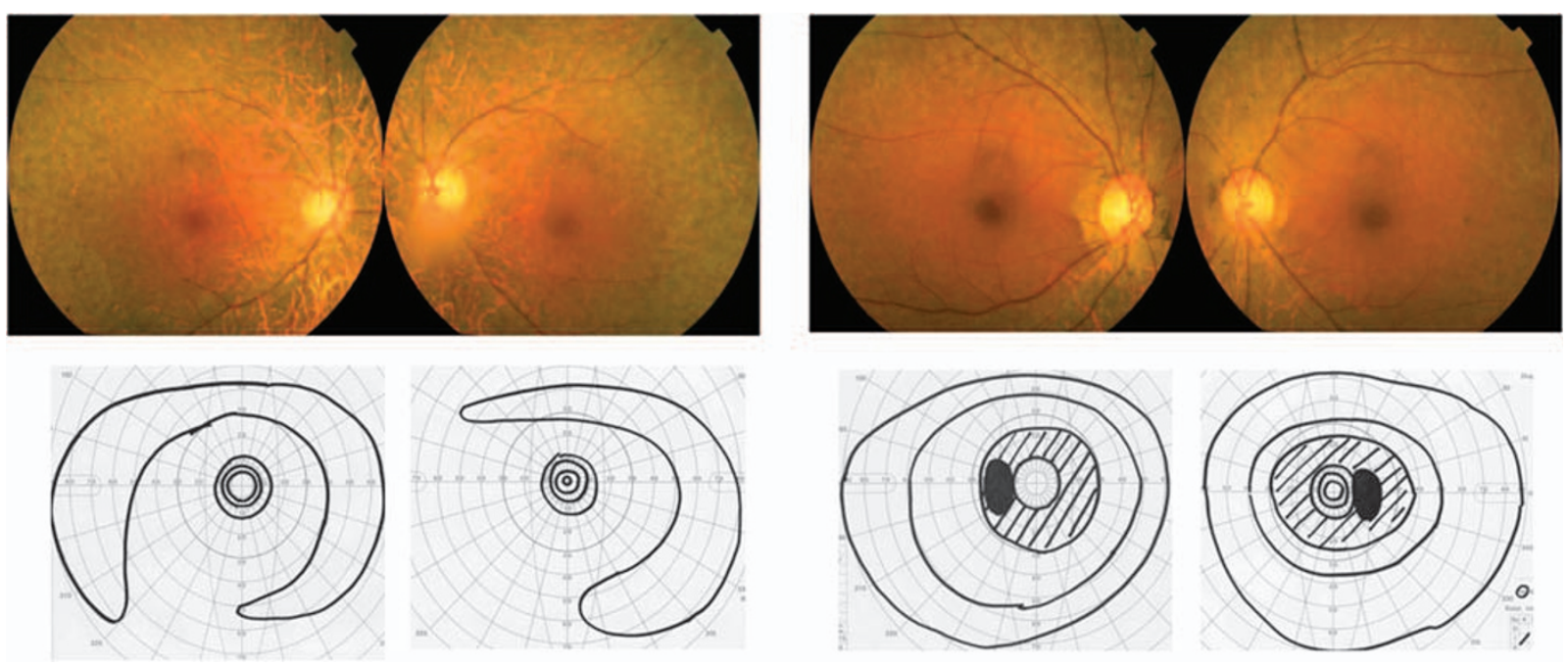

Figure 2 Fundus photographs and visual fields of two retinitis pigmentosa (RP) patients. (left) Case 1 was a 72-year-old man whose best-corrected visual acuity was 20/20 in both eyes and had a composite score of 48.9. (right) Case 2 was a 62-year-old woman whose best-corrected visual acuity was 20/20 in both eyes and had a composite score of 90.3 .

Table 1 Mean composite NEI VFQ-25 scores of 10 subscales in RP patients and normal controls

\begin{tabular}{llcr}
\hline Subscales & RP patients $(\mathrm{n}=40)$ & Control $(\mathrm{n}=40)$ & $P$ \\
\hline General vision & $59.4 \pm 17.4(20-80)$ & $80.0 \pm 12.0(60-100)$ & $<0.001$ \\
Near vision & $67.1 \pm 22.5(0-100)$ & $92.0 \pm 11.2(66.7-100)$ & $<0.001$ \\
Distance vision & $67.8 \pm 17.4(0-87.5)$ & $90.8 \pm 10.2(83.3-100)$ & $<0.001$ \\
Peripheral vision & $40.1 \pm 22.5(0-75)$ & $92.5 \pm 12.9(50-100)$ & $<0.001$ \\
Colour vision & $87.5 \pm 18.8(0-100)$ & $96.9 \pm 8.4(75-100)$ & 0.0027 \\
Ocular pain & $77.1 \pm 22.5(37.5-100)$ & $74.4 \pm 18.3(50-100)$ & 0.4184 \\
Role limitation & $73.1 \pm 25.1(25.0-100)$ & $88.8 \pm 11.9(48.3-100)$ & 0.0052 \\
Dependency & $73.4 \pm 23.1(16.7-100)$ & $97.7 \pm 7.1(75-100)$ & $<0.001$ \\
Social function & $75.4 \pm 20.1(37.5-100)$ & $95.6 \pm 8.3(75-100)$ & $<0.001$ \\
Mental health & $60.7 \pm 22.4(12.5-100)$ & $91.7 \pm 13.1(75-100)$ & $<0.001$ \\
\hline
\end{tabular}

NEI VFQ-25, National Eye Institute Visual Function Questionnaire-25; RP, retinitis pigmentosa.

Numbers represent mean \pm standard deviation. Numbers in parentheses indicate range of scores.

composite scores and the degree of visual field loss was calculated by using Spearman's rank correlation test. A $P<0.05$ was considered statistically significant.

\section{Results}

The fundus photographs and visual fields from a 72-year-old RP patient (case 1, left) and a 62-year-old RP patient (case 2, right) are shown in Figure 2. The visual field loss was graded as grade 4 in case 1 and grade 2 in case 2 , although the visual acuity was $20 / 20$ in both patients. It is interesting to note that the 10-subscale composite scores were markedly lower in case 1 (48.9) than in case 2 (90.3).

The mean composite scores for the 10-subscale items in the RP patients and controls are shown in Table 1. There was no difference in the ocular pain score between RP patients and controls. The 10-subscale composite scores ranged from 32.1 to 89.3 (mean: $68.4 \pm 15.0$ ) in the RP patients, and from 82.6 to 100 (mean: $90.1 \pm 7.4$ ) in the controls. This difference between the RP patients and controls was significant $(P=0.00004)$. For the individual scores, the differences between the RP patients and controls were significant in all subscales except ocular pain.

The correlation between visual acuity and the 10-subscale composite scores was not significant ( $r=0.016, P=0.922$; Figure 3 ), but there was a negative significant correlation between the grade of visual field defect and the 10-subscale composite scores $(r=-0.519$, $P=0.0006$; Figure 4 ). The mean grade of visual field defect was $2.7 \pm 1.9$ (range: $0-6$ ) in RP patients. In all 40 RP patients, the grades of visual field loss were the same in both eyes. 


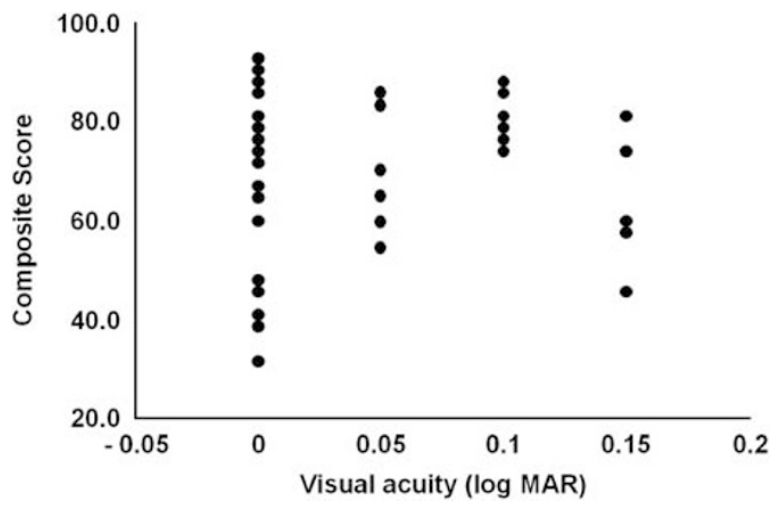

Figure 3 Relationship between the visual acuity in logMAR units and the 10-subscales composite score in retinitis pigmentosa (RP) patients. The correlation between the two factors is not significant $(r=0.016, P=0.922)$.

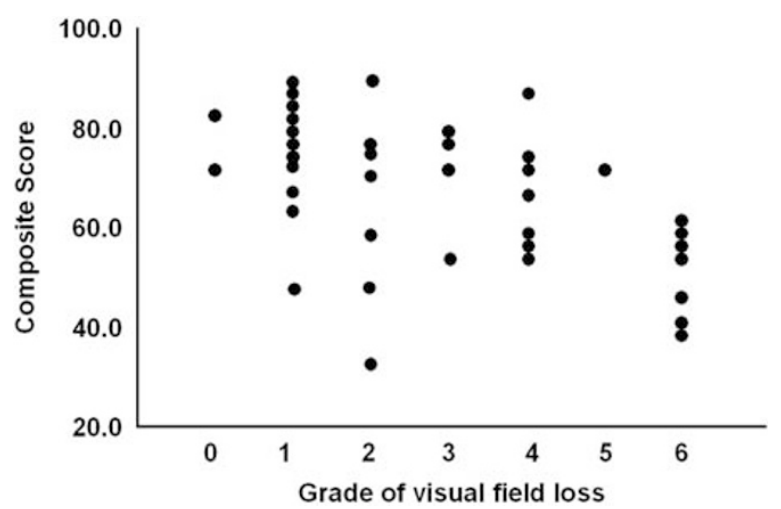

Figure 4 Relationship between the grade of visual field loss and the 10-subscales composite score in retinitis pigmentosa (RP) patients. There is a significant negative correlation between the two factors $(r=-0.519, P=0.0006)$.

\section{Discussion}

We used the NEI VFQ-25 questionnaire to evaluate the VRQOL in RP patients and found that the composite scores were significantly lower in RP patients than in the normal controls except for ocular pain. In interpreting these results, we need to consider the socioeconomic differences between RP patient and controls because some of the subscales and composite score in the control were better than in the control of Suzukamo study. ${ }^{14}$ However, there were similar differences between the RP patient and control of Suzukamo data when we used the data of the control in Suzukamo paper.

It has been reported that the average composite scores of the NEI VFQ-25 in Japanese population was 66.0 for patients with cataract, 69.8 in glaucoma, 51.0 in AMD, and 80.1 in normal individuals. ${ }^{14}$ The composite score of the RP patients was comparable to those with cataract and glaucoma, but much higher than those with AMD. There are several factors, which might account for this difference in the QOL composite score. First, the demographics of patients in the AMD study most likely differed from that of the RP patients. Second, patients with AMD lose their central vision and maintain their peripheral visual field, whereas RP patients maintain their central vision and lose their peripheral field. And third, the change in vision is slow and progressive in RP patients, whereas it is relatively rapid in AMD patients. The lower score in the AMD patients suggests that the loss of central vision affects the VRQOL more than the loss of the peripheral vision in RP patients. However, further studies will be needed to determine the real cause of the differences in VRQOL in AMD patients and RP patients.

We found a significant negative correlation between the VRQOL and the degree of peripheral visual field loss. Even with the same good vision, there were some variations of the VRQOL depending on the visual field loss using the NEI VFQ-25. Similar findings were reported by Szlyk et $a l^{1}$ that the visual field area of RP patients calculated by computerized perimetry was correlated with the patients' self-assessments of daily activities. The correlation was higher for visual acuity for their RP patients than our result because Szlyk et al did not exclude patients with poor visual acuity.

However, it has also been reported that using the total area of the visual field was not appropriate because the total area may be distorted by large peripheral visual fields compared with the smaller central visual field..$^{18,19}$ Therefore, we modified the classifications of visual field loss in RP patients used by other investigators, and classified them into seven grades. Furthermore, we did not use both eyes for visual field loss evaluations. Previous studies suggest that the binocular visual field testing is a better way to examine the relationship between visual field defect and quality of life. ${ }^{10,20}$ Further studies of a large number of RP cases will be needed to evaluate the effect of visual field loss on the VRQOL.

The classification of peripheral visual field loss in RP is difficult because the progression of visual field loss is not the same among RP patients. Heckenlively ${ }^{17}$ reported that at the early stage, there is a slight loss in the superior peripheral field and scotomatous areas in the midequatorial field. At the intermediate stage, the many scotomatous areas become more confluent so that a partial to full-ring scotoma emerges. As the disease advances, the superior and nasal fields are lost, leaving a central island of field with elongated temporal islands. Grover $e t a l^{18}$ proposed five types of visual field defects for RP patients from a ring scotoma to residual central area without peripheral islands. Iijima et al ${ }^{19}$ also proposed four types of visual field defects. According to 
Iijima's classification, type 1, visual field constriction mild with normal peripheral limits in at least one sector; type 2, visual field moderately constricted with central field larger than $15^{\circ}$; type 3 , central field less than $15^{\circ}$ with peripheral field island; and type 4, visual field extremely constricted with only a central field of less than $15^{\circ}$. We modified these reported classifications to evaluate smaller changes in the visual fields.

In spite of this, there was some variability in the composite scores among patients with the same grade of visual field loss. Szlyk et $a l^{3}$ reported that some RP patients with severe vision loss could have minimal impairment in carrying out everyday life tasks. It is possible that the psychological factors may also have a strong influence on self-assessment. Hahm et al ${ }^{11}$ reported that RP patients with depression had poorer vision-related functions compared with those patients without depression. Szlyk et al also reported that selfreported score was significantly correlated with the actual task performance assessed by a low-vision specialist, and that the assessment of actual performance validated the use of self-reports in RP patients.

There are at least three following limitations to our study: first, we excluded RP patients whose visual field did not match any of the grades of our classification; second, we studied only patients whose visual acuity was better than 0.7 in decimal units; and third, RP patients are not a homogenous group. In addition, the number of RP patients was relatively small.

In conclusion, our findings demonstrate that the vision-related QOL measured with the NEI VFQ-25 is significantly correlated with the degree of peripheral field loss in RP patients whose central vision was preserved. Thus, these results indicate that the grade of the visual field defect is a better predictor of the QOL than the visual acuity in typical RP patients.

\section{References}

1 Szlyk JP, Fishman GA, Alexander KR, Revelins BI, Derlacki DJ, Anderson RJ. Relationship between difficulty in performing daily activity and clinical measures of visual function in patients with retinitis pigmentosa. Arch Ophthalmol 1997; 115: 53-59.

2 Sumi I, Matsumoto S, Okajima O, Shirato S. The relationship between visual disability and visual scores in patients with retinitis pigmentosa. Jpn J Ophthalmol 2000; 44: 82-87.

3 Szlyk JP, Seiple W, Fishman GA, Alexander KR, Grover S, Mahler CL. Perceived and actual performance of daily tasks: Relationship to visual function tests in individuals with retinitis pigmentosa. Ophthalmology 2001; 108: 65-75.

4 Mangione CM, Lee PP, Pitts J, Gutierrez P, Berry S, Hays RD. Psychometric properties of the National Eye institute Visual
Function Questionnaire (NEI-VFQ). Arch Ophthalmol 1998; 116: 1496-1504.

5 Mangione CM, Lee PP, Gutierrez PR, Spritzer K, Berry S, Hays RD. Development of the 25-item National Eye Institute Visual function Questionnaire. Arch Ophthalmol 2001; 119: 1050-1058.

6 Klein R, Moss SE, Klein BE, Gutierrez P, Mangione CM. The NEI-VFQ-25 in people with long-term type 1 diabetes mellitus: The Wisconsin Epidemiologic Study of Diabetic Retinopathy. Arch Ophthalmol 2001; 119: 733-740.

7 Schiffman RM, Jacobsen G, Whitcup MS. Visual fuctioning and general health status in patients with uveitis. Arch Ophthalmol 2001; 119: 841-849.

8 Deramo VA, Cox TA, Syed AB, Lee PP, Fekrat S. Vision-related quality of life in people with central retinal vein occlusion using the 25-item National Eye Institute Visual Function Questionnaire. Arch Ophthalmol 2003; 121: 1297-1302.

9 Cahill MT, Banks AD, Stinnett SS, Toth CA. Vision-related quality of life in patients with bilateral severe age-related macular degeneration. Ophthalmology 2005; 112: 152-158.

10 Varma R, Wu J, Chong K, Azen SP, Hays RD. Impact of severity and bilaterally of visual impairment on healthrelated quality of life. Ophthalmology 2006; 113: 1846-1853.

11 Hahm BJ, Shin YW, Shim EJ, Jeon HJ, Seo JM, Chung H et al. Depression and the vision-related quality of life in patients with retinitis pigmentosa. Br J Ophthalmol 2008; 92: 650-654.

12 Rossi GC, Milano G, Tinelli C. The Italian version of the 25-item National Eye Institute Visual Function Questionnaire: Translation, validity, and reliability. J Glaucoma 2003; 12: 213-220.

13 Nordmann JP, Viala M, Sullivan K, Arnould B, Berdeaux G. Psychometric Validation of the National Eye Institute Visual Function Questionnaire-25 (NEI VFQ-25) French version: In a population of patients treated for ocular hypertension and glaucoma. Pharmacoeconomics 2004; 22: 197-206.

14 Suzukamo Y, Oshika T, Yuzawa M, Tokuda Y, Tomidokoro A, Oki K et al. Psychometric properties of the 25-item National Eye Institute Visual Function Questionnaire (NEI VFQ-25), Japanese version. Health Qual Life Outcomes 2005; 3: 65-75.

15 Burstedt MS, Monestam E, Sandgren O. Associations between specific measures of vision and vision-related quality of the life in patients with Bothnia dystrophy, a defined type of retinitis pigmentosa. Retina 2005; 25: 317-323.

16 Williams GP, Pathak-Ray V, Austin MW, Lloyd AP, Millington IM, Bennett A. Quality of life and visual rehabilitation: An observational study of low vision in three general practices in West Glamorgan. Eye 2007; 21: 522-527.

17 Heckenlively JR, Krauss HR. Visual fields in retinitis pigmentosa. In: Heckenlively JR (ed.). Retinitis Pigmentosa. J.B. LIPPINCOTT Company: Philadelphia, 1988, pp 25-36.

18 Grover S, Fishman GA, Anderson R, Alexander KR, Derlacki DJ. Rate of visual field loss in retinitis pigmentosa. Ophthalmology 1997; 104: 460-465.

19 Ijima H, Okajima O, Okammoto M, Hirato T. Stage of visual field change in pigmentary retinal dystrophy. Folia Ophthalmologica Japonica 1986; 37: 448-450.

20 Jampel HD, Friedman DS, Quigley H, Miller R. Correlation of the binocular visual field with patients assessment of vision. Invest Ophthalmol Vis Sci 2002; 43: 1059-1067. 